\section{Music inspired Newton's rainbow}

Isaac Newton was among the great scientists who took inspiration from music (see Nature 519, 262; 2015). In fact, music drove him to add two new colours to the rainbow.

The medieval rainbow had just five colours: red, yellow, green, blue and violet. Newton added two more - orange and indigo - so that the colours would be "divided after the manner of a Musical Chord" (I. Newton in Opticks 4th edn, 127 (William Innys, 1730); see also K. McLaren Color Res. Application 10, 225-229; 1985).

On a seemingly unrelated note, Ed Hawkins and colleagues make a plea to scrap rainbow colour scales in scientific graphics (Nature 519, 291; 2015). They warn that these palettes can "introduce false perceptual thresholds in the data".

It was Newton's perception that first introduced new colour thresholds, although these were subjective rather than false. It gives the lie to the old saying that artists see what they believe, but scientists believe what they see. Len Fisher University of Bristol, $U K$.

len.fisher@bristol.ac.uk

\section{Disputed start dates for Anthropocene}

As members of the

Anthropocene Working Group, we contend that the proposed new geological epoch should reflect a unique stratigraphic unit that is characterized by unambiguous, widespread and essentially permanent anthropogenic signatures in rock, glacial ice or marine sediments. We therefore find the two dates chosen by Simon Lewis and Mark Maslin to be questionable candidates for the start of the Anthropocene (Nature 519, 171-180; 2015).

For the first date suggested by the authors, the short-lived

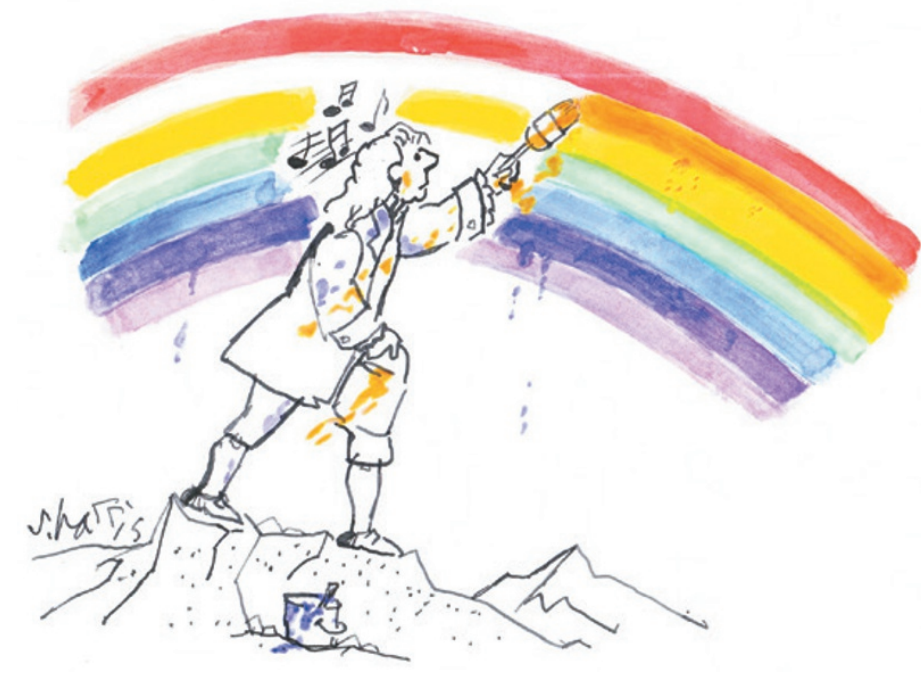

decline of atmospheric carbon dioxide that reached its minimum in 1610 is not an ideal stratigraphic marker for an epoch-scale boundary. It is one small dip of several in the Holocene epoch, which began about 11,700 years ago, and is not outside the range of natural variability - in contrast to the signature associated with industrialization. Associated indicators of colonization of the Americas, such as the worldwide spread of pollen from maize (corn), lasted for centuries and so do not represent nearsynchronous markers.

By the time of the authors' other suggested date of 1964 , the 'great acceleration' in human activity was well under way (W. Steffen et al. Anthropocene Rev. 2, 81-98; 2015). Also, the year 1964 is later than the near-synchronous upward inflections of many physical and socio-economic trends and their respective stratigraphic signals, which date to around 1950 (J. Zalasiewicz et al. Quat. Int. http://doi.org/zjf; 2015)

We need further comprehensive analyses of the advantages and limitations of different proposed markers before we can arrive at an effective starting date for the Anthropocene.

Jan Zalasiewicz ${ }^{\star}$ University of Leicester, UK. jaz1@leicester.ac.uk
Confederation of Open Access Repositories can support research institutions in storing their data. National data services are already providing generic support to researchers (see, for example, go.nature.com/uns6zy). Now, different fields need to converge on common formats for data storage and preservation if such measures are to be effective. Andrew Gonzalez McGill University, Montreal, Canada. Pedro R. Peres-Neto University of Quebec in Montreal, Canada. andrew.gonzalez@mcgill.ca

\section{China needs more monitoring apps} go.nature.com/3z9oju for full list).

\section{Act to staunch loss of research data}

Never before have scientists had the ability to generate and collect so much data - recent estimates suggest that the global scientific output is doubling roughly every decade (see L. Bornmann and R. Mutz, preprint at http://arxiv. org/abs/1402.4578v3; 2014, and go.nature.com/nzejwh). It is alarming, therefore, that the odds of data being lost are estimated to increase by $17 \%$ in every year after publication (T. H. Vines et al. Curr. Biol. 24, 94-97; 2014). And this does not include the $80 \%$ or so of research data that are inaccessible or unpublished (B. P. Heidorn Libr. Trends 57, 280-299; 2008).

Information is lost when researchers fail to store, archive or share their data, for example, and as a result of ageing technology or corruption of data-storage devices. A culture of systematic data curation is needed to stem this loss, but it is not yet in place across research fields - even though curation costs a fraction of the funding used to generate the data in the first place. Standardized protocols would ensure that data are shared and properly curated worldwide.

Global networks such as the
There are more than one billion mobile devices in China, offering huge potential for citizen scientists to contribute to a cleaner and safer environment. The scientific community should rapidly develop mobile apps to collect and monitor environmental and biodiversity data.

In one example of how China's citizen science could take off, journalist Jing Chai showed how to use a mobile app to follow air quality in the local environment as part of her 'Under the Dome' documentary on smog pollution, which was viewed millions of times in just 24 hours (see go.nature.com/2hj7wa).

Better apps would allow China's 8,000 or so environmental nongovernmental organizations to tap into the massive volume of data collected by professionals and citizens. Also, collaborations among all three groups could improve and streamline data management and analysis.

Government financial support for citizen science is currently sparse. Greater investment by large agencies such as the National Natural Science Foundation of China would quickly reap rewards. Jian Zhang Aarhus University, Denmark.

Xiaolei Huang Fujian Agriculture and Forestry University, China. jian@bios.au.dk 